\title{
Controlled release of 5-fluorouracil from microporous zeolites
}

Marios Spanakis ${ }^{\mathrm{a}}$, Nikolaos Bouropoulos ${ }^{\mathrm{b}}$, Dimitrios Theodoropoulos ${ }^{\mathrm{c}}$, Lamprini

Sygellou $^{\mathrm{d}}$, Sinead Ewart ${ }^{\mathrm{e}}$, Anastasia Maria Moschovi ${ }^{\mathrm{d}}$, Angeliki Siokou ${ }^{\mathrm{d}}$, Ioannis

Niopas $^{\mathrm{a}}$, Kyriakos Kachrimanis ${ }^{\mathrm{c}}$, Vladimiros Nikolakis ${ }^{\mathrm{f}}$, Paul A. Cox ${ }^{\mathrm{e}}$, Ioannis S. Vizirianakis $^{\mathrm{a}}$, Dimitrios G. Fatouros ${ }^{\mathrm{c}, *}$

${ }^{a}$ Aristotle University of Thessaloniki, School of Pharmacy, Department of Pharmacology, GR-54124 Thessaloniki, Greece

${ }^{\mathrm{b}}$ Department of Materials Science, University of Patras, 26504 Rio, Patras, Greece

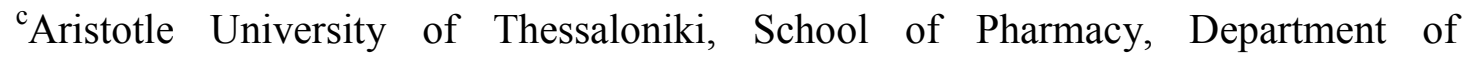
Pharmaceutical Technology, GR-54124 Thessaloniki, Greece

${ }^{\mathrm{d}}$ Foundation for Research and Technology, Hellas-Institute of Chemical Engineering Sciences - FORTH/ICE-HT, P.O. Box 1414, GR-26504 Patras, Greece

${ }^{\mathrm{e}}$ School of Pharmacy and Biomedical Sciences, University of Portsmouth, St. Michael's Building, White Swan Road, Portsmouth PO1 2DT, UK

${ }^{\mathrm{f}}$ University of Delaware, Catalysis Center for Energy Innovation \& Department of Chemical and Biomolecular Engineering ,150 Academy St., Newark, DE 19716, USA

${ }^{*}$ Corresponding author: Dr Dimitrios G. Fatouros

e-mail:dfatouro@pharm.auth.gr

Tel: +302310997653

Fax: +302310997653 
Manuscript Word Count: 4892 words

Abstract Word Count: 150 words

Number of Figures/Tables: 6/1, Number of References: 18 


\begin{abstract}
Zeolite particles with different pore diameter and particle size were loaded with the model anticancer drug 5-fluorouracil. The loaded zeolites were characterized by means of SEM, XRD, DSC, XPS, $\mathrm{N}_{2}$ physisorption and FT-IR. Higher loading of 5FU was observed for NaX-FAU than BEA. Release studies were carried out in simulated gastric fluid. Release of 5-FU from NaX-FAU showed exponential-type behaviour with the drug fully released within $10 \mathrm{~min}$. In the case of BEA, the kinetics of 5-FU shows a multi-step profile with prolonged release over time. Molecular dynamics simulations showed that diffusion of the drug molecule through the BEA framework is lower than for NaX-FAU due to increased van der Waals interaction between the drug and the framework. The effect of zeolitic particles on the viability of Caco-2 monolayers showed that the NaX-FAU particles cause a reduction of cell viability in a more pronounced way compared with the BEA particles.
\end{abstract}

Keywords: zeolites, controlled release, molecular modeling, cytocompatibility. 


\section{Background}

Zeolites are microporous materials with regular pore architectures and compositions with cages and channels running through them. ${ }^{1,2}$ Their properties, such as ionexchange, separation, and catalysis, and their roles as hosts in nanocomposite materials, are essentially determined by their unique structural characteristics, such as the size of the pore window, the accessible void space, the dimensionality of the channel system, and the numbers, sites and types of extra framework cations. ${ }^{2}$ The dimensions of these pores, channels and cages are such that drug molecules can be accommodated inside them. ${ }^{3-7}$ Moreover, the drug molecules are likely to diffuse out of the channel systems slowly, thus controlling their release rate. In a previous study we investigated the effect of different loading procedures on the release profile of the active compound. ${ }^{7}$ In an attempt to investigate the effect of different types of zeolites on the release kinetics and the physical state of the active compound, the anticancer drug 5-fluorouracil (5-FU) was encapsulated into NaX-FAU and BEA zeolitic particles. These two zeolites differ in framework type, pore diameter, and particle size. Specifically, the BEA framework type belongs to the tetragonal crystal system having an 3-dimentional channel 12 member ring pore system comprising straight and sinusoidal channels along the <100> and [001] with 6.6 × $6.7 \AA$ and 5.6 × $5.6 \AA$ openings respectively, however the maximum diameter of a sphere that can diffuse along its channels for all three crystallographic directions is $5.95 \AA$. NaX-FAU belongs to the cubic system containing supercages with $\sim 13 \AA$ that communicate through $\sim 7.35 \AA$ windows. Additionally the effect of Si/Al ratios $\sim 250$ for BEA; less than 1.5 for NaX-FAU and the hydrophobicity of zeolitic particles (BEA zeolite is more hydrophobic compared to NAX-FAU ) to the encapsulation and release of 5-FU were further assessed. 
5-FU is an anticancer agent poorly absorbed with variations in bioavailability ranging between 0 and $80 \%$. On the other hand parenteral administration of FU, it is rapidly eliminated with an apparent terminal half-life of approximately 8-20 min. ${ }^{8}$

In view of the promise of zeolites as carriers via the oral route of administration, we have characterized zeolitic particles loaded with 5-FU by means of scanning electron microscopy (SEM), $\mathrm{N}_{2}$ physisorption, powder x-ray diffraction (XRD), differential scanning calorimetry (DSC), X-ray photoelectron spectroscopy (XPS) and fouriertransform infrared spectroscopy (FT-IR). Their cytotoxicity was assessed by means of (thiazolyl blue tetrazolium bromide) MTT assay to Caco-2 cell lines. The release profiles of the loaded particles were evaluated in simulated gastric fluid. Finally these studies were complemented by molecular dynamic calculations.

\section{Methods}

\section{Materials}

Molecular sieves, $13 \mathrm{X}$ powder, $\sim 2 \mu \mathrm{m}$ average particle size were purchased from Sigma-Aldrich. All purchased chemicals were used as received without further purification. 5-FU was obtained from Sigma-Aldrich. The synthesis and characterisation by means of Scanning Electron Microscopy and $\mathrm{N}_{2}$ physisorption studies of BEA zeolitic nanoparticles is described in SI.

\section{Preparation of drug loaded zeolites}

$200 \mathrm{mg}$ of zeolite was impregnated in $30 \mathrm{~mL}$ of an ethanolic containing $80 \mathrm{mg}$ of 5 FU under constant stirring at room temperature for $24 \mathrm{~h}$. The solvent was removed by filtration through MF-Millipore ${ }^{\mathrm{TM}}$ membrane filters, pore size $0.05 \mu \mathrm{m}$ for BEA and $0.45 \mu \mathrm{m}$ for NaX-FAU zeolites respectively and then dried at $40^{\circ} \mathrm{C}$ for $24 \mathrm{~h}$. The loading efficiency of the zeolite particles was determined by adding $10 \mathrm{mg}$ of loaded samples in $20 \mathrm{~mL}$ EtOH and stirred for $24 \mathrm{~h}$. The samples were centrifuged in a 
Heraeus Labofuge $400 \mathrm{R}$ at $4000 \mathrm{rpm}$ for $30 \mathrm{~min}$. The supernatant was collected and determined with a UV-1700 spectrophotometer Shimadzu at $266 \mathrm{~nm}$.

\section{Characterization of the zeolite loaded particles}

The zeolite loaded particles were characterized by means of XRD, FTIR, DSC and XPS (For details see SI).

\section{Stability studies of zeolitic particles in dissolution medium}

The effect of the medium on the zeolite NaX-FAU framework was determined as reported previously with little modification. Briefly, $50 \mathrm{mg}$ of zeolite particles were incubated in $20 \mathrm{~mL}$ of $\mathrm{HCl} 0.1 \mathrm{M} \mathrm{pH} 1.2$ for 5 and $120 \mathrm{~min}$ respectively. The samples were centrifuged in a Heraeus Labofuge $400 \mathrm{R}$ at $4000 \mathrm{rpm}$ for $30 \mathrm{~min}$. The supernatants were collected and dried at $40^{\circ} \mathrm{C}$ for $24 \mathrm{~h}$ and further analyzed by means of SEM and XRD.

\section{Molecular modeling - Dissolution studies}

Molecular dynamics (MD) simulations were performed using the program Materials Studio version 6.0 and drug release from loaded zeolites samples was studied by using a USP/Ph.Eur. paddle dissolution apparatus (Pharma Test PT-DT7). All release studies were carried out under sink conditions in triplicate. (For details see SI).

\section{Cell viability - MTT assay - Transepithelial electrical resistance (TEER) studies}

Viability of Caco-2 cells was evaluated using the MTT assay based on the ability of viable cells to convert thiazolyl blue tetrazolium bromide solution to the blue formazan crystals in their mitochondria. ${ }^{9}$ Also, by recording the formulation-induced changes in transepithelial electrical resistance (TEER), the use of Caco-2 monolayers as an in vitro probe of the potential permeation-enhancing capacity of the applied formulation has been employed. ${ }^{10}$ (For details see SI). 


\section{Results}

\section{Characterization of zeolitic particles}

The textural properties of the zeolitic particles are illustrated at Table S1 (SI). Scanning electron microscopy studies revealed that the NaX-FAU particles exhibited multi-faceted spherulite crystals with a mean diameter of $1.98 \pm 0.30 \mu \mathrm{m}$ as determined by digital image analysis (Figure 1A, Table S1, SI). ${ }^{11}$ On the contrary the BEA particles had a mean diameter of $140 \pm 47 \mathrm{~nm}$ (Figure 1B, Table S1, SI). The $\mathrm{N}_{2}$ physisorption isotherm in the BEA and NaX-FAU crystals (Figure 1C and 1D) corresponds to a type I isotherm according to the IUPAC classification.

\section{Characterization of the drug loaded particles}

The X-ray diffractograms of all samples are shown in Figure 2A. The loaded samples exhibit the characteristic reflections of NaX-FAU and BEA zeolites, respectively, indicating that the encapsulation procedure did not destroy the zeolite structure.

Specifically for the drug-loaded NaX-FAU diffractograms, a change in relative intensities of the (220) and (311) diffraction peaks before and after the encapsulation can be seen (Figure 1A), while all reflections show a slightly increased intensity. This observation might be due to the presence of drug molecules in the zeolite pore network or on the surface. Moreover, the presence of a characteristic peak, attributed to the (200) Miller plane of 5-FU at $27.5^{\circ}$, in the NaX-FAU loaded sample indicates the presence of crystalline drug. In the case of BEA, the XRD pattern (Figure 2A) is dominated by the peaks associated with BEA, with a notable absence of any peaks associated with 5-FU, suggesting an absence of crystalline 5-FU.,11

The FTIR spectra of pure 5-FU, drug loaded zeolites and empty zeolites are shown in Figure 2B. Residual ethanol in the samples was not detected as evident from Figure 2B. In the case of pure drug the characteristic bands at 1667 and $1247 \mathrm{~cm}^{-1}$ are 
assigned to the overlapped stretching vibration absorption of $\mathrm{C}=\mathrm{O}, \mathrm{C}=\mathrm{C}$ and $\mathrm{C}-\mathrm{N}$ respectively. Absorption at $1435 \mathrm{~cm}^{-1}$ is due to in-plane bending vibration of $\mathrm{C}-\mathrm{H}$ in $(-\mathrm{CF}=\mathrm{CH}-)$ and at $813 \mathrm{~cm}^{-1}$ is assigned to out-of-plane bending vibration absorption of $\mathrm{C}-\mathrm{H}$ in $(-\mathrm{CF}=\mathrm{CH}-)$. The band at $1725 \mathrm{~cm}^{-1}$ is assigned to stretching vibrations of $\mathrm{C}=\mathrm{O}$ and $\mathrm{N}-\mathrm{H}$ in cyclic imide $(\mathrm{CO}-\mathrm{NH}-\mathrm{CO}){ }^{12,13}$ It is apparent that characteristic bands of 5-FU are recognized in the spectrum of NaX-FAU loaded samples suggesting that that the drug is associated with the zeolitic NaX-FAU microparticles. An additional interesting observation is that in all FTIR spectra, a broad absorption band in the range of $3000-3800 \mathrm{~cm}^{-1}$ is present in all zeolite samples (both empty and loaded), which can be attributed to the presence of strongly bound crystal water, and indicates that the loading treatment has not significantly affected the hydration state of the zeolites.

In the DSC thermograms, Figure $2 \mathrm{C}$, no sign of melting attributable to the drug is seen, which indicates that the drug does not exist in crystalline form. The only prominent feature of the DSC thermograms, is a broad endotherm in the region of 40 $150{ }^{\circ} \mathrm{C}$ for BEA, but extends up to $250{ }^{\circ} \mathrm{C}$ for the loaded NaX-FAU.

The spatial distribution of 5-FU on zeolitic particles was probed by means of XPS via its $\mathrm{F}$ and $\mathrm{N}$ atoms.

The wide spectra of NaX-FAU empty and NaX-FAU loaded samples which show that In, O , C , Al, Si , Na are present on the surface are depicted in Figure 3A. Small peaks of N1s and F1s are also present giving evidence for the presence of the drug in the zeolites. The wide scan spectra of BEA empty and BEA loaded samples, which show In, O, C and Si are illustrated in Figure 3B. The F1s and N1s peaks can be also traced for the loaded sample, but their presence is more apparent in the high resolution scans. 
The atomic ratio for the 5-FU is found to be 0.47 , in excellent agreement with the nominal value which is 0.50 . For the NAX-FAU loaded sample only the area of the F1s peak that corresponds to the drug, at binding energy $688.5 \mathrm{eV}$, was taken into account (Figure 3A) and from this the F:N surface atomic ratio was found to be 0.56 . For the loaded BEA F:N=0.39. These values are tabulated in Table 1 together with the surface atomic ratios $\mathrm{N}: \mathrm{Si}$ and F:Si for the empty and loaded samples.

Overall the physicochemical characterization of the loaded zeolitic particles give evidence of the presence of 5-FU in both NaX-FAU and BEA zeolites. Bearing in mind that XPS is a surface sensitive spectroscopy obtaining about $40 \%$ of its signal from the first 2 or $3 \mathrm{~nm}$, the small intensity of the F1s and N1s peaks for the loaded BEA sample could imply that 5-FU is located mainly in the pore structure of BEA zeolites. The higher intensity of these peaks for the loaded NAX-FAU indicates a more homogenous distribution of 5-FU in the zeolite's channels and possible presence on its outer surface as well. (The XPS spectra of pure 5-FU and the N1s XPS peaks and F1s XPS peaks are available in SI, Figures S1A, S1B, S1C and S1D respectively).

\section{Encapsulation efficiency, release studies and molecular dynamics}

The drug loading studies revealed significantly higher encapsulation efficiency for the NaX-FAU zeolite $16.18 \pm 1.18 \%(\mathrm{w} / \mathrm{w})$ compared to BEA zeolite $6.02 \pm 0.66 \%$ $(\mathrm{w} / \mathrm{w})$ respectively. The higher amount to the NaX-FAU zeolite could be attributed higher loadings in the pore due to differences in pore size or geometry and possibly to the stronger interactions of the 5-FU with the pore walls of $\mathrm{NaX}-\mathrm{FAU}$ particles compared to BEA particles.

The release profiles of 5-FU from NaX-FAU and BEA particles are shown in Figure 4A. The results show marked differences in the release profiles observed for the two zeolites used. A steep release of the drug from $\mathrm{NaX}-\mathrm{FAU}$ particles was noticed. After 
3 min $c a .83 \%$ of the initial amount the drug was released. Release from NaX-FAU shows exponential-type behaviour and is relatively rapid with the drug fully released within approximately 10 minutes. There is no evidence from the release profile to suggest that diffusion from within the internal channel system of the framework is any different to that from the surface.

On the contrary, in the case of BEA the release of 5-FU shows a multi-step profile, with about $15 \%$ of the drug being released in a distinct exponential step in the first 10 minutes, another $40 \%$ being released in a subsequent step in 40 minutes, while the remaining amount is released within an hour. The overall release of the drug is much slower, and takes about twelve times longer to complete than for NaX-FAU.

The effect of the acidic environment of the medium on the zeolite showed that the later is presumably the dominant factor rather than the medium for the release of the active compound. (SI Figures S2 and S3).

The dissolution data were fitted to zero-order, first-order, Korsmeyer-Peppas, Higuchi, Hixson - Crowell and Weibull models. Dissolution models were fitted using the DDSolver add-in program for Microsoft Excel. ${ }^{14}$

The determination coefficient $\left(\mathrm{r}^{2}\right)$ was used to indicate the best fit model. The release rate constants were calculated from the slope of the various plots (Table S2).

The in vitro drug release from zeolites was best explained by Weibull model, as the plots showed the highest linearity $\left(r^{2}=0.988\right)$ for NaX-FAU and $\left(r^{2}=0.997\right)$ for BEA zeolites respectively. Weibull plots can describe the dissolution curve in terms of applicable parameters. The shape parameter, $\beta$, characterizes the curve as either exponential $(\beta=1)$, s-shaped with upward curvature followed by a turning point $(\beta>1)$, or with a steeper initial slope than is consistent with the exponential $(\beta<1)$. In the case of $\mathrm{NaX}$-FAU zeolites the $\beta$ parameter was 0.292 consistent with a steep release of 5- 
FU as evident from the release profile in Figure 4A. In the case of BEA zeolite the $\beta$ parameter was $>1(2.415)$ exhibiting an s-shaped curvature as illustrated in Figure 4A.

The NaX-FAU particles are significantly larger so their external (geometrical) surface area is smaller than that of BEA. The micropore pore volumes and surface areas are similar; therefore it could be assumed that in the case of NaX-FAU the existence of the $\sim 1.3 \mathrm{~nm}$ super cages or higher Al content allow the encapsulation of larger amounts of 5-FU. It is also anticipated that loading depends on the number of $\mathrm{Na}$ sites per unit cell (assuming that the interaction with the cation is one of the main reason that can cause adsorption). In that case the $\mathrm{Si} / \mathrm{Al}$ ratio of BEA is $\sim 250$ which corresponds to 1 cation every $\sim 4$ unit cells. On the other hand NaX-FAU usually has $\mathrm{Si} / \mathrm{Al}<1.5$ meaning that numerous $\mathrm{Na}$ are available per unit cell. To make a quantitative comparison we need to make some normalization. However since each zeolite has a different number of $\mathrm{Si}, \mathrm{Al}$ atoms in the unit cell, but considering the vast differences in $\mathrm{Si} / \mathrm{Al}$ ratio a qualitative argument seems justified. In addition the nature of the zeolites might affect the release of the drug. For instance NaX-FAU is very hydrophilic so 5-FU might be released quickly because water molecules can be accommodated in the pores as well. On the contrary low Al content BEA is more hydrophobic repelling water molecules from the pores. The results showed that the release of 5-FU from NaX-FAU particles is rapid and that any molecules located inside the internal channel system are not released significantly slower than those on the surface. However, release of 5-FU from BEA particles is prolonged by those molecules absorbed inside the BEA channel system. In a recent study the release of 5-FU from zeolites with different $\mathrm{Si} / \mathrm{Al}$ ratios was studied in physiological medium. ${ }^{15}$ 
The results shown that the aluminum content was critical as the release of the drug was higher when the $\mathrm{Si} / \mathrm{Al}$ ratio was increased.

The molecular dynamics calculations support this interpretation of the experimental data. The molecular dynamics studies revealed that 5-FU displays significantly different diffusion rates for 5-FU molecules located inside the internal pore system of the two zeolites. The plots of root mean square (RMS) displacement against time are shown in Figure 4B. In both cases, an upward slope is observed which shows that the drug is able to move through both zeolite frameworks at the temperature of the simulations $(310 \mathrm{~K})$. The gradient obtained for $\mathrm{NaX}-\mathrm{FAU}$ is significantly steeper than the one observed for BEA. On this basis, it would be anticipated that the drug molecule would diffuse in and out of the NaX-FAU structure much more easily than through BEA. This also implies that the drug should load inside the NaX-FAU structure more readily. Both of these predictions are consistent with the experimental observations. The dimensions of the largest pores in BEA, along the $<100>$ direction, are approximately $6.6 \times 6.7 \AA$ whereas the windows between the cages along the $<111>$ direction in NaX-FAU are slightly larger at 7.4 x $7.4 \AA$. The average nonbonded interaction between the drug and the zeolite framework was $-25.0 \mathrm{kcal} \mathrm{mol}^{-1}$ in the BEA structure but significantly less, $-16.0 \mathrm{kcal} \mathrm{mol}^{-1}$ in NaX-FAU. This difference is dominated by the increased van der Waals' interaction between 5-FU and the BEA structure where the interaction is $-23.3 \mathrm{kcal} \mathrm{mol}^{-1}$ compared with -15.7 $\mathrm{kcal} \mathrm{mol}^{-1}$ in NaX-FAU. Electrostatic interactions between both frameworks and the drug have a much smaller difference, $-0.3 \mathrm{kcal} \mathrm{mol}^{-1}$ for NaX-FAU and $-1.7 \mathrm{kcal} \mathrm{mol}^{-1}$ for BEA and so we can attribute the difference in the diffusion rates to the 'tighter fit' of the 5-FU molecule in the BEA channel system which gives rise to much stronger short-range van der Waals interactions. The molecular dynamic simulations show 
that the increased interaction between 5-FU and BEA is not strong enough to prevent the molecule from diffusing along the zeolite's channel, but that it is enough to exert a significant degree of controlled release. In contrast, movement of the drug through the NaX-FAU framework is much less hindered by the interaction between the internal walls of the zeolite structure and the molecule is able to diffuse in and out of NaXFAU much faster. The faster diffusion of 5-FU through NaX-FAU can also been seen in Figure 4D. In NaX-FAU, the drug molecule moves rapidly within the cages of the structure before moving to the next cage via one of the interconnecting windows. Residence time in the NaX-FAU cages is relatively small and several hops are made from cage to cage per 1000 ps, making diffusion relatively fast (Figure 4D). In contrast, over the same time period, 5-FU diffuses relatively slowly through the pore channel system of BEA, where the movement is much more constrained by the close 'fit' of the molecule inside the internal channels, (Figure 4C).

\section{Proliferative activity studies at Caco-2 cell monolayers}

Cytotoxicity studies for zeolites are rather scarce. ${ }^{6,7,16,}$ Cultured human intestinal epithelium is regarded a useful model for the study of oral drug absorption in that it allows a preliminary assessment of the permeability, toxicity, metabolism and transport of potential therapeutic agents. ${ }^{17}$

Cell growth, (expressed as cells $/ \mathrm{cm}^{2}$ versus time), for the empty zeolite particles exhibited similar pattern to control-untreated Caco-2 cell cultures (Figure 5A \& B). At $3 \mathrm{~h}$ no significant difference was noticed between the control and the free drug, empty zeolites and drug loaded samples either for BEA or NaX-FAU respectively (ANOVA, $\mathrm{p}>0.05)$.

In the absence of drug, alterations in cell growth were noticed after $8 \mathrm{hr}$ incubation with particles. Empty BEA particles at all concentrations tested showed no toxicity 
compared to the control (ANOVA, p>0.05). On the contrary free drug and 5-FU loaded particles showed higher toxicity compared to the control (ANOVA, $\mathrm{p}<0.05$ ). In the case of NaX-FAU particles, all the other formulations were significantly different compared to control (ANOVA, $\mathrm{p}<0.05$ ) with the exception of empty NaXFAU particles at low concentrations $(0.01 \mathrm{mg} / \mathrm{mL})$.

The cell growth significantly decreased (ANOVA, p<0.05) for all NaX-FAU formulations compared to the control at $24 \mathrm{~h}$. However, when BEA formulations were tested the reduction of cell growth as compared to the control was minimal (ANOVA, $\mathrm{p}>0.05$ ) for 0.1 and $0.01 \mathrm{mg} / \mathrm{mL}$ of empty zeolites respectively. After further incubation up to $48 \mathrm{hr}$ in culture, the cells exhibited a small delay in reaching the plateau phase of cell growth. This effect on Caco-2 cell proliferation capacity has also been verified by calculating the half maximal inhibitory concentration $\left[\mathrm{IC}_{50}\right]$ values for 24 and/or $48 \mathrm{~h}$, respectively (Table S3, SI).

There was no direct relation between the textural properties (e.g. BET values) of the zeolitic particles and cell viability. However the morphology of the particles might play a role in the cell growth. The multi-faceted NaX-FAU microparticles might penetrate cell membranes more easily compared with the almost spherical BEA nanoparticles. Finally, the size of the particles seems to affect the cell growth as the bigger NaX-FAU microparticles can induce higher toxicity to the cells compared to BEA nanoparticles. Previously toxicity studies of silicalite-1 nanoparticles with different crystal size (from 30 up to $500 \mathrm{~nm}$ ) and surface functionalisation to human embryonic kidney 293 (HEK-293) cells and RAW264.7 macrophage cell lines showed that cell toxicity is type dependent. ${ }^{17}$ Template-free zeolites loaded with mitoxantrone tested in prostate cancer cells (PC-3) and mammary carcinoma cells (MCF-7) respectively. Empty zeolites showed no toxicity to both cell lines up to 0.5 
$\mathrm{mg} / \mathrm{mL}$. When the drug was loaded, higher toxicity was exhibited compared with the empty ones. However lower toxicity values were obtained for drug loaded zeolites compared with the free drug.

When 5-FU was loaded into zeolite particles, similar toxicity values were obtained as compared with that of free 5-FU (Figure 5 A \& B). Importantly, in both cases, the loaded particles seem to alter the cell growth behavior in a dose-dependent manner. Moreover, the same trend was observed for drug-loaded particles and the 5-FU alone as evident from data shown in Figure 4 A \& B.

In broad agreement with previous studies, ${ }^{18}$ the $\mathrm{IC}_{50}$ values of 5-FU were estimated to $0.0252 \mathrm{mg} / \mathrm{mL}(24 \mathrm{~h})$ and $0.0239 \mathrm{mg} / \mathrm{mL}(48 \mathrm{~h})$, respectively. The $\mathrm{IC}_{50}$ values for empty zeolites were significantly higher $(\mathrm{t}$-test $\mathrm{p}<0.05)$ compared to free drug for both 24 and $48 \mathrm{~h}$ (Table S2). On the contrary, drug-loaded zeolites being pharmacological active exhibited significantly lower (t-test $\mathrm{p}<0.05) \mathrm{IC}_{50}$ values compared with the empty ones. It has to be noted that significantly lower $\mathrm{IC}_{50}$ values were calculated for drug-loaded NaX-FAU particles compared to BEA-loaded particles ( $\mathrm{t}$-test $\mathrm{p}<0.05)($ Table $\mathrm{S} 2)$. The latter difference might be explained from the release profiles of the drug where a fast release of 5-FU was observed from NaX-FAU zeolite (Figure 5A) allowing a more timely higher concentration of the drug to exhibit its cytotoxic activity faster compared to BEA-loaded particles.

\section{Effect of zeolitic particles on TEER values of Caco-2 cells}

Tight junctions serve as barriers to paracellular diffusion and the measurement of the transepithelial resistance (TEER) is believed to be a good indication of the tightness of the junctions between epithelial cells. ${ }^{10}$ Incubation of intestinal epithelial cells (Caco-2) with different BEA and NaX-FAU particles in concentrations of $0.01,0,1$ and $1 \mathrm{mg} / \mathrm{mL}$ resulted in an immediate reduction of TEER values at $3 \mathrm{~h}$ up to $20 \%$ for 
all concentrations (Figure $6 \mathrm{~A}$ and $\mathrm{B}$ ). The highest reduction in TEER values was measured at a concentration of $1 \mathrm{mg} / \mathrm{mL}$ of BEA and $\mathrm{NaX}-\mathrm{FAU}$ particles at $24 \mathrm{~h}$ thus the reduction in TEER is concentration-dependent.

This reduction was statistically different from that shown with $0.01 \mathrm{mg} / \mathrm{mL}$ for all time points for the NaX-FAU particles (t-test $\mathrm{p}<0.05$ ). In the case of BEA particles it was significantly different at 3,8 and 24 h there was respectively. With removal of the zeolitic particles (indicated by an arrow at both graphs of Figure 6 (A \& B) and substitution of the apical medium with fresh medium) reversibility of the effects was noticed. The monolayers started to recover slowly and a constant increase in resistance, toward the initial values was found. A comparison of the effect of the size of the particles on the TEER across Caco- 2 cell monolayers revealed that the $\mathrm{NaX}$ FAU particles which are bigger have the tendency to induce a higher decrease to the TEER values (not significantly, t-test $\mathrm{p}>0.05$ ). This final increase might be due to a reorganisation of junctional-complexes after they have been damaged by the exposure to zeolitic particles. From TEER recovery data obtained $24 \mathrm{~h}$ post-removal of the particles, it can be hypothesized that zeolites are not likely to affect cell viability. The TEER values are in accordance with the low toxicity of the zeolitic particles as evident from the MTT assay. The reduction of TEER values in the presence of zeolitic particles suggest their propensity to increase possible the paracellular permeability of active compounds.

\section{Discussion}

The two zeolite structures investigated in this study have different pore architectures and exhibit markedly different release profiles for the same drug. Careful selection of the appropriate drug/framework combination is therefore crucial in determining the optimum system for drug delivery. The molecular dynamics method suggests that the 
slower release rate observed for BEA can be attributed to the increased van der Waals' interaction between the drug and the host framework. This increased interaction is not large enough to prevent diffusion of the drug, but is sufficient to exert a considerable degree of controlled release. The agreement between the simulations and the observed release profiles suggests that molecular dynamics is a highly promising tool for screening zeolite/drug combinations prior to experimental investigation. Finally, cytocompatibility studies show that zeolitic particles are nontoxic to human cells in vitro underlining their significant potential for in vivo applications. 


\section{REFERENCES}

1. Baerlocher S, McCusker LB., Olsen DH. Atlas of Zeolite Framework Types. $6^{\text {th }}$ Edition, Elsevier Science: Amsterdam 2007.

2. Cundy CS, Cox PA. The hydrothermal synthesis of zeolites: history and development from the earliest days to the present time Chem. Rev. 2003; 103: $663-702$.

3. Dyer A, Morgan S, Wells P, Williams C. The use of zeolites as slow release anthelmintic carriers. J. Helmin. 2000; 74: 137 - 141.

4. Horcajada P, Marquez-Alvarez C, Ramila A, Perez-Pariente J, Vallet-Regi, M. Controlled release of Ibuprofen from dealuminated faujasites Solid State Sci. 2006; 8: 1459 - 1465.

5. Grund S, Doussineau T, Fischer D, Mohr GJ. Mitoxantrone-loaded zeolite beta nanoparticles: preparation, physico-chemical characterization and biological evaluation J. Colloid Inter. Sci. 2012; 365: 33 - 40.

6. Datt A, Fields D, Larsen SC. An Experimental and Computational Study of the Loading and Release of Aspirin from Zeolite HY. J. Phys. Chem. C. 2012; 116 : 21382- 21390

7. Fatouros DG, Douroumis D, Nikolakis V, Ntais S, Moschovi AM, Trivedi V, Khima B, Roldo M, Nazar H, Cox PA. In vitro and in silico investigations of drug delivery via zeolite BEA. J. Mater. Chem. 2011; 21: 7789 - 7794.

8. Diasio RB, Harris BE. Clinical pharmacology of 5-fluorouracil. Clin. Pharmacokinet. 1989; 16: 215-237.

9. Mosmann T. Rapid colorimetric assay for cellular growth and survival: application to proliferation and cytotoxicity assays J. Immunol. Methods 1983; $65: 55-63$ 
10. Makhlof A, Werle M, Tozuka Y, Takeuchi H. A mucoadhesive nanoparticulate system for the simultaneous delivery of macromolecules and permeation enhancers to the intestinal mucosa J. Contr. Rel. 2011; 149 : 81 88.

11. Shen S.-C, Ng WK, Chia L, Dong Y.-C, Tan RBH. Stabilized amorphous state of ibuprofen by co-spray drying with mesoporous SBA-15 to enhance dissolution properties J. Pharm. Sci. 2010; 99 : 1997 - 2007.

12. Huang L, Sui W, Yuanxiu Wang Y, Jiao Q. Preparation of chitosan/chondroitin sulfate complex microcapsules and application in controlled release of 5-fluorouracil. Carbohyd. Polym. 2010; 80 : 168 - 173.

13. Lin FH, Lee YH, Jian CH, Wong JM, Shieh MJ, Wang CY. A study of purified montmorillonite intercalated with 5-fluorouracil as drug carrier. Biomaterials. 2002; 23 : 1981 - 1987.

14. Zhang Y, Huo M, Zhou J, Zou A, Li W, Yao C, Xie S. DDSolver: An add-in program for modelling and comparison of dissolution profiles. AAPS J., 2010; $12: 263-271$.

15. Datt A, Burns EA, Dhuna NA, Larsen SC. Loading and release of 5fluorouracil from $\mathrm{HY}$ zeolites with varying $\mathrm{SiO}_{2} / \mathrm{Al}_{2} \mathrm{O}_{3}$ ratios. Microp. Mesop. Mater. 2013, 167, $182-187$

16. Petushkov A, Intra J, Graham JB, Larsen SC, Salem AK. Effect of crystal size and surface functionalization on the cytotoxicity of silicalite-1 nanoparticles Chem. Res. Toxicol. 2009; 22 : 1359 - 1368.

17. Artursson P. Epithelial transport of drugs in cell culture. I: A model for studying the passive diffusion of drugs over intestinal absorptive (Caco-2) cells J. Pharm. Sci. 1990; 79 : 476 - 482. 
18. Ciccolini J, Peillard L, Evrard A, Cuq P, Aubert C, Pelegrin A, Formento P, Milano G, Catalin J. Enhanced antitumor activity of 5-fluorouracil in combination with 2'-deoxyinosine in human colorectal cell lines and human colon tumor xenografts.” Clin Cancer Res. 2000; 6 :1529 - 1535. 


\section{FIGURE LEGENDS}

Figure 1 : The SEM images of the zeolite particles. (Insets) histograms representing statistical size distribution of the zeolite particles were generated from SEM image analysis for (A) BEA and (B) NaX-FAU particles. The $\mathrm{N}_{2}$ physisorption isotherms of (C) BEA and (D) NaX-FAU particles.

Figure 2: (A) x-ray diffraction patterns of pure NaX-FAU and BEA zeolites and their complexes (B) FT-IR spectra of pure NaX-FAU and BEA zeolites and their complexes. (C) DSC thermograms of pure NaX-FAU and BEA zeolites and their complexes.

Figure 3: X-ray photoelectron spectroscopy (XPS) spectra of (A) of NAX-FAU empty and NAX-FAU loaded surfaces and (B) BEA empty and BEA loaded surfaces

Figure 4: (A) Release profiles of 5-FU from BEA and NaX-FAU particles in simulated gastric fluid. The error bars indicated are mean \pm S.D. $(n=3)$. (B) Rootmean displacement graphs for 5-FU inside the zeolite beta (BEA) framework and faujisite (NaX-FAU). Diffusion of 5-FU through the channel system of (C) BEA and (D) NaX-FAU. The location of the drug molecule is shown every 10 ps during the first 1000 ps of the simulations.

Figure 5: Caco-2 cell viability at 3, 824 and $48 \mathrm{~h}$ after incubation with (A) BEA and (B) NaX-FAU zeolitic particles empty and loaded with 5-FU. The data are given as mean \pm S.D. $(n=3)$. 
Figure 6 : Application of zeolite particles at increasing concentrations (\% w/v) on Caco-2 cell intestinal epithelia at $\mathrm{pH} 7.4$ and their effect on the TEER, $(\mathrm{n}=3)(\mathbf{A})$ BEA, (B) NaX-FU particles respectively. (The arrows indicate that samples removed and cells washed at $24 \mathrm{~h}$ ). 
FIGURE 1
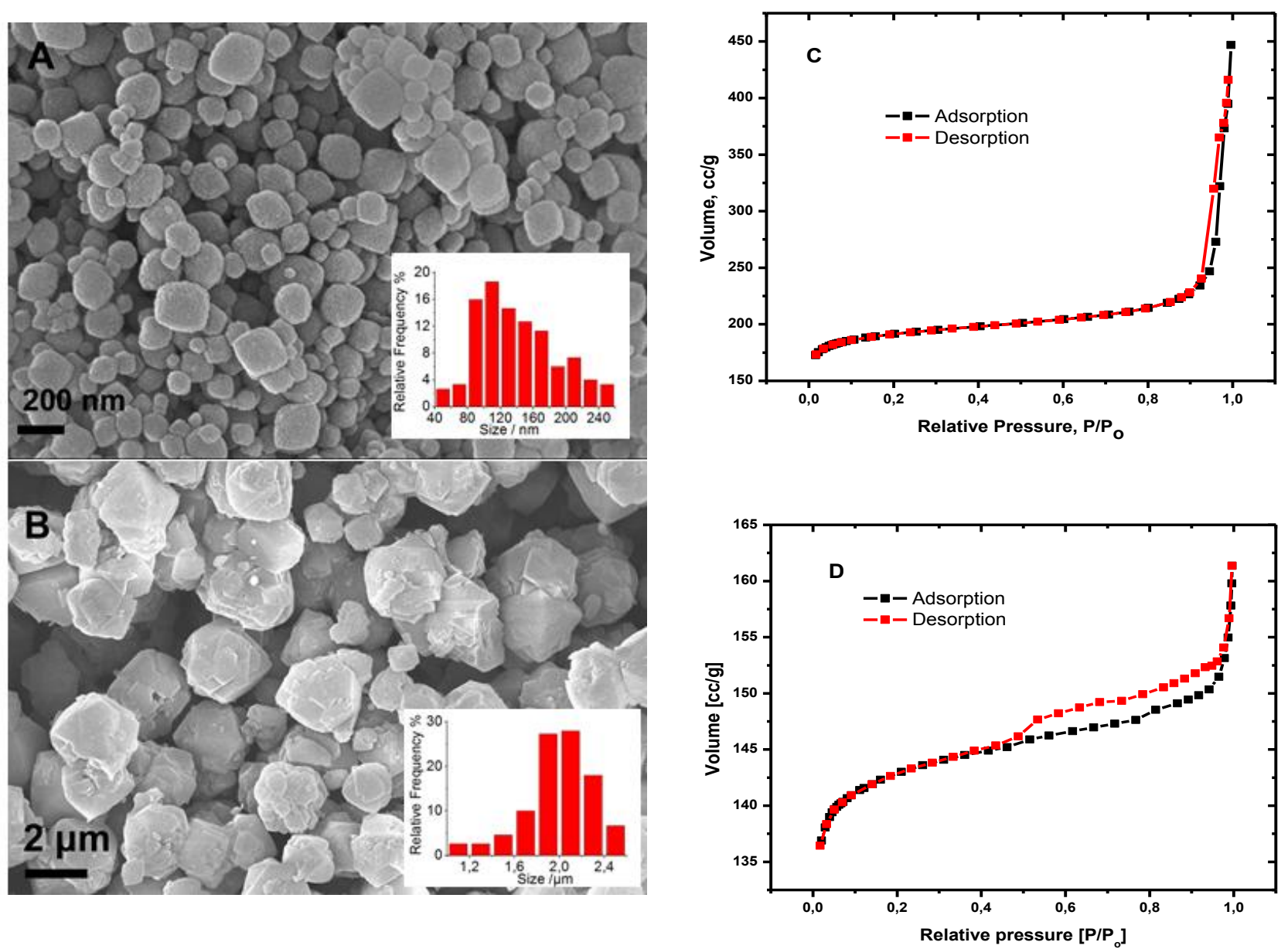
FIGURE 2
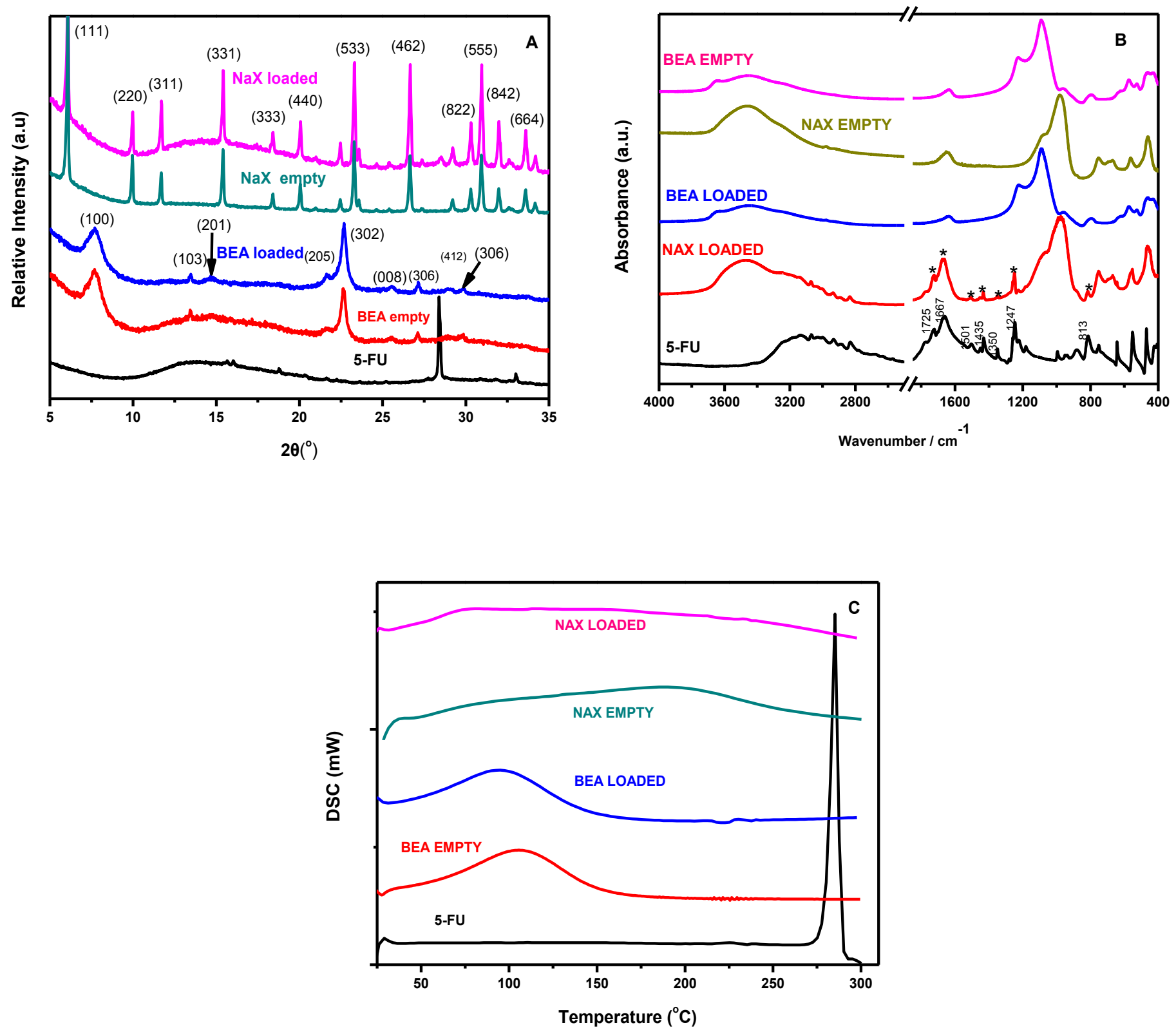
FIGURE 3
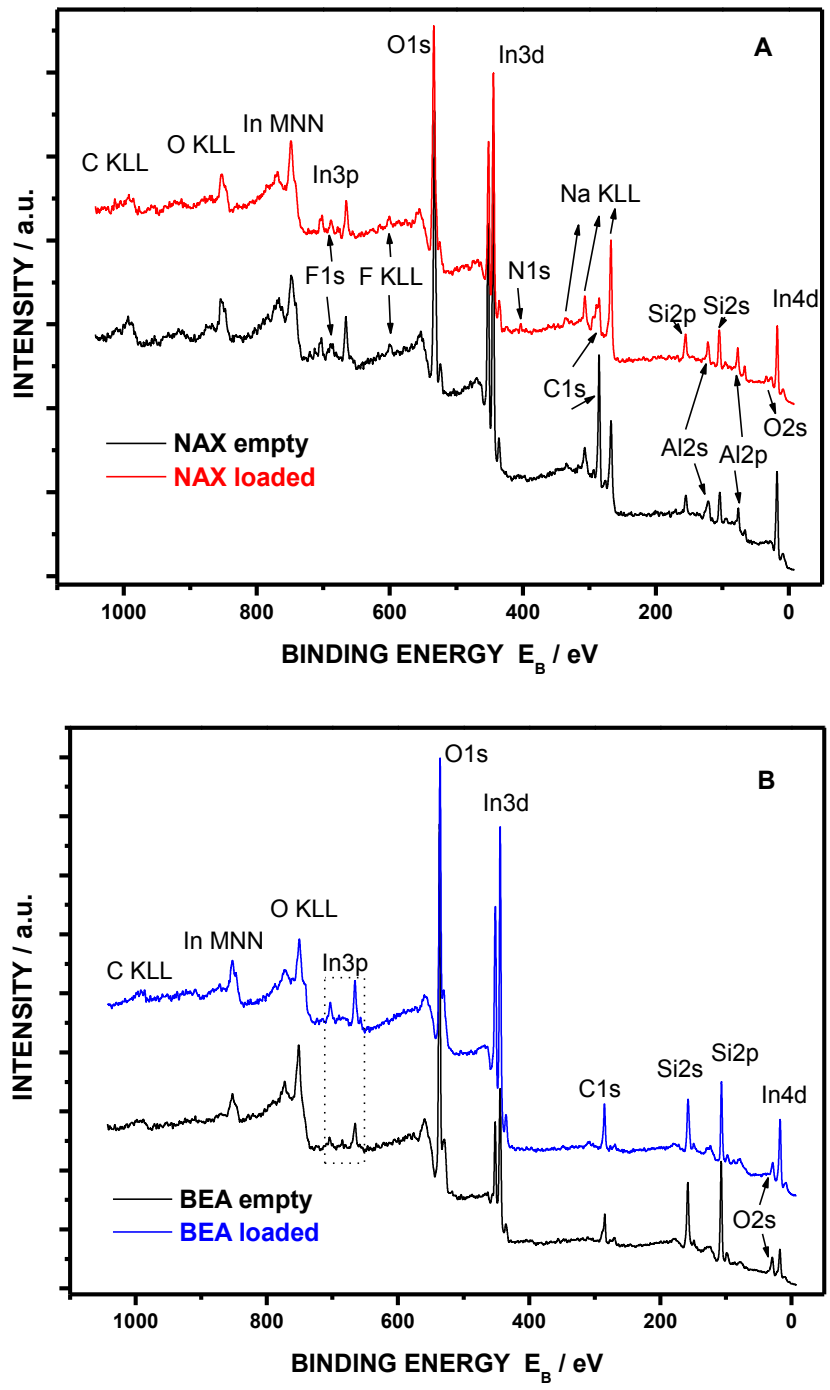
FIGURE 4
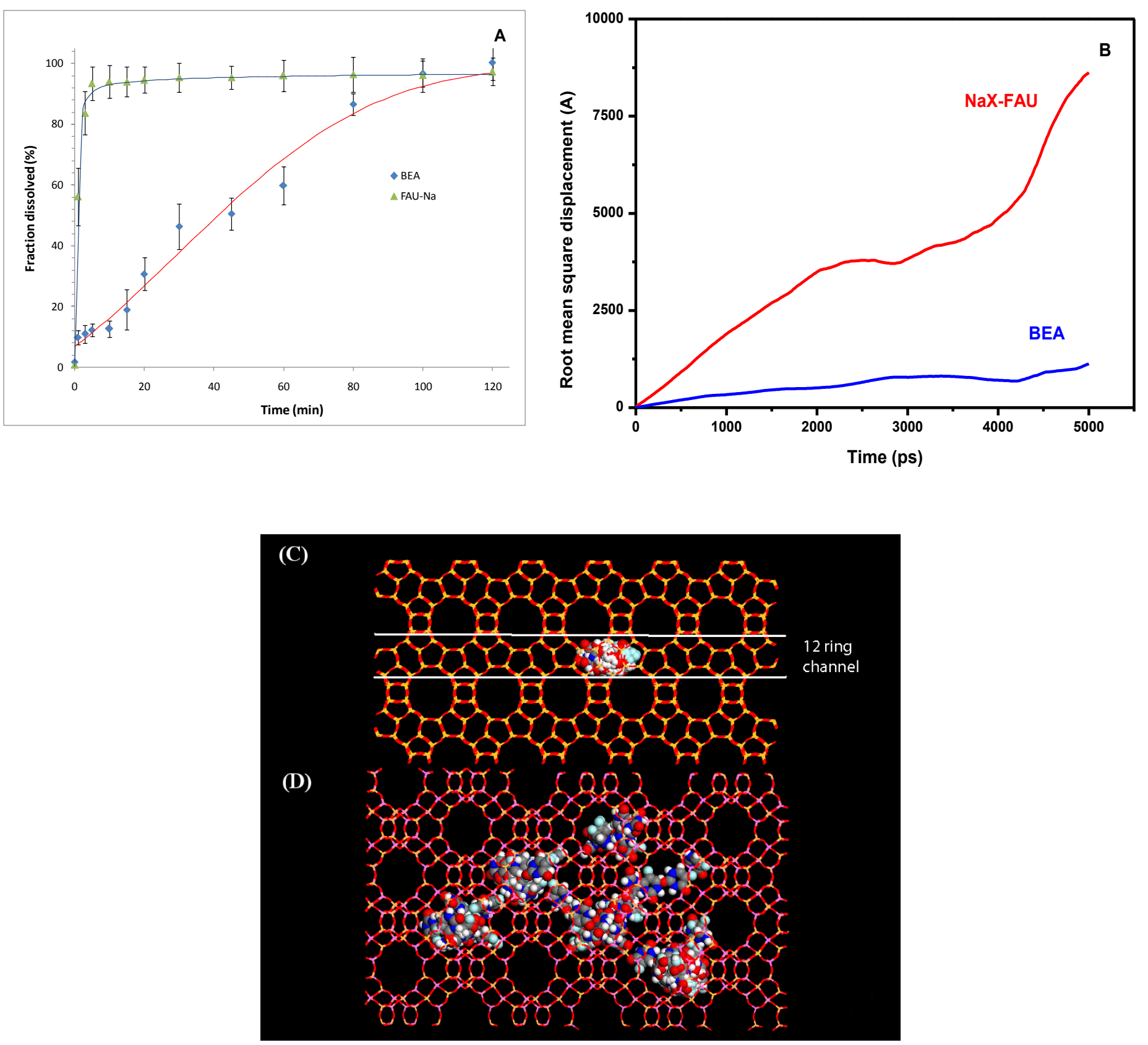
FIGURE 5
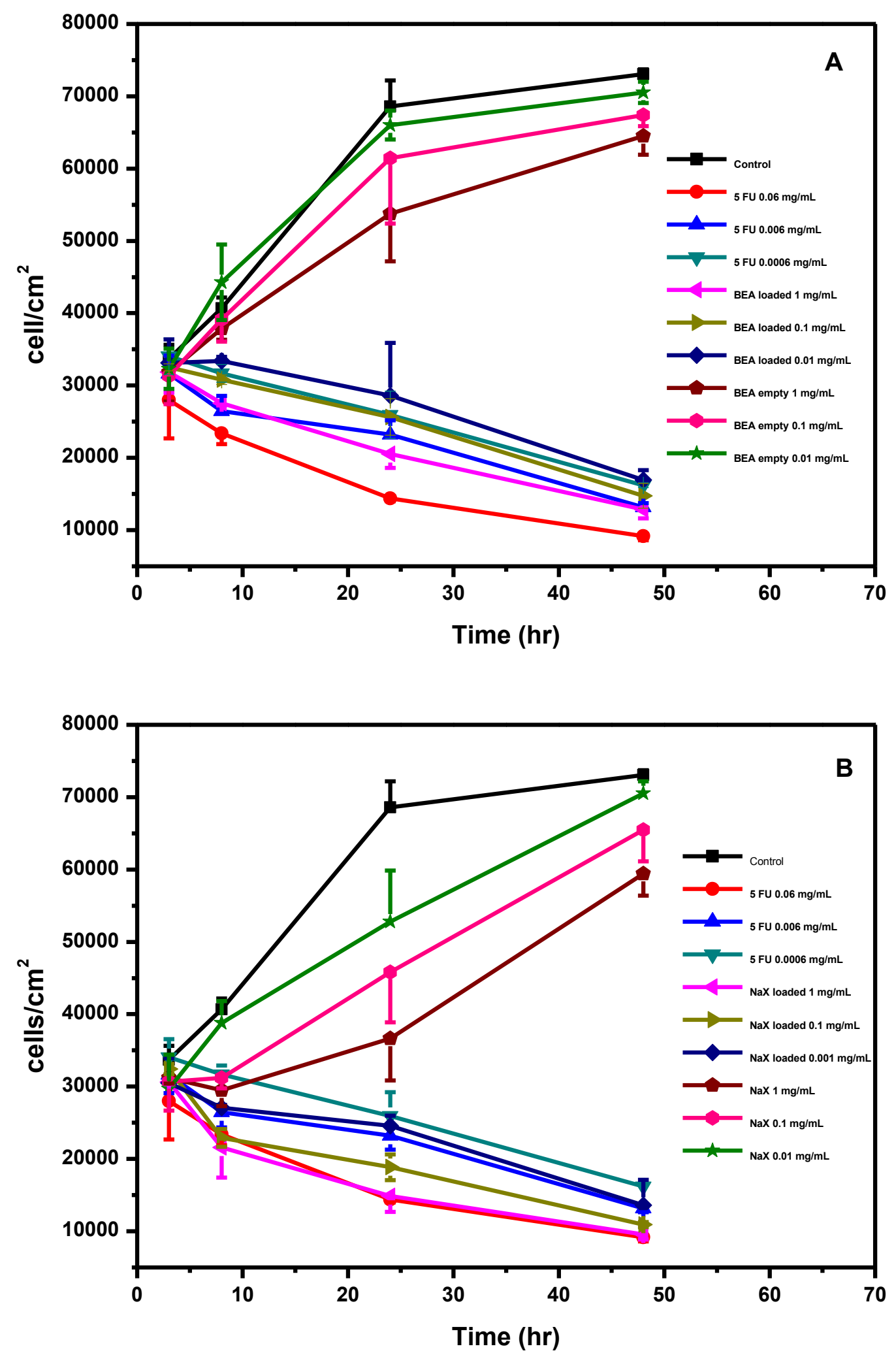
FIGURE 6
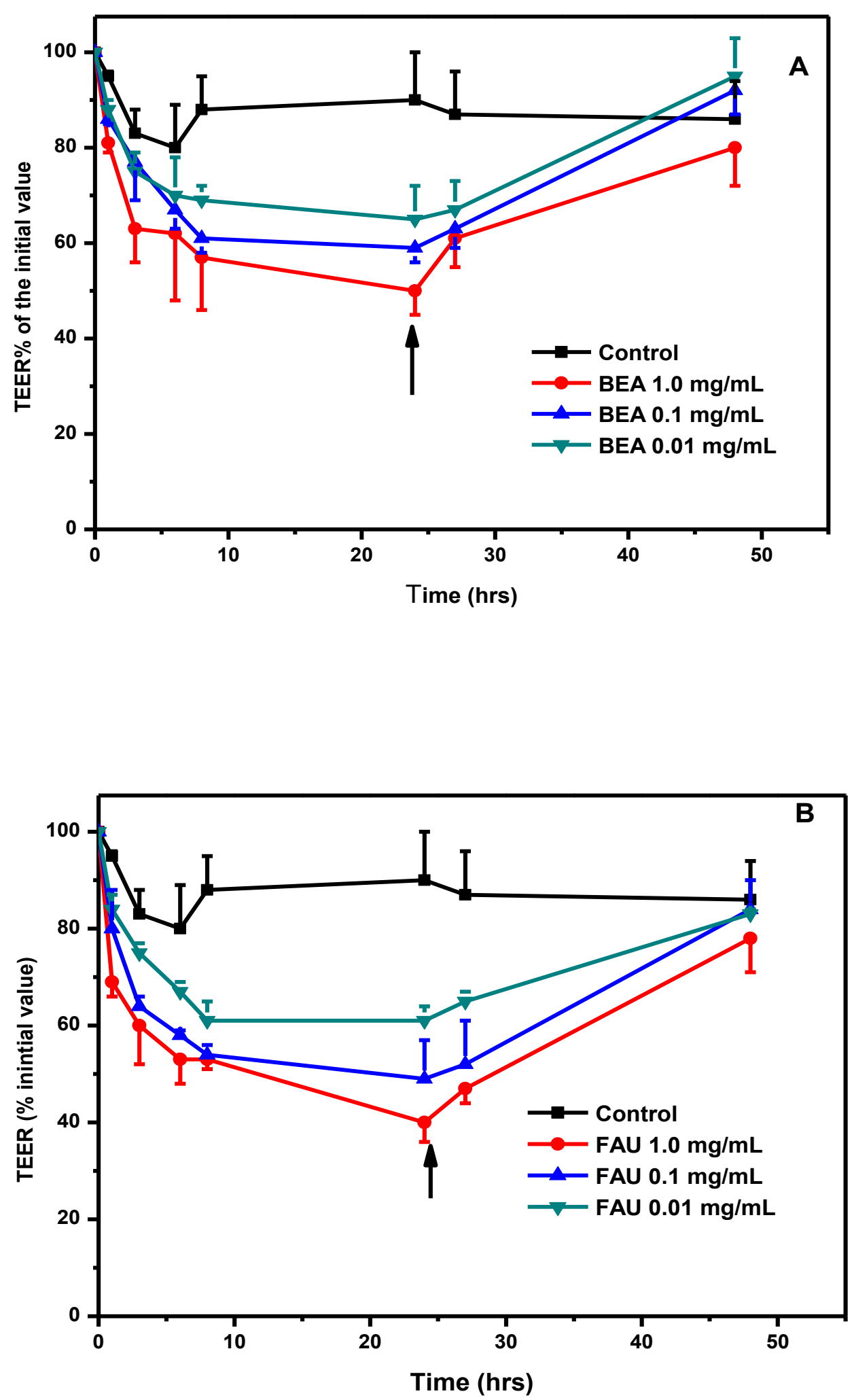
Table 1: Surface atomic ratios for 5-FU and for the empty and loaded zeolites.

\begin{tabular}{lccc}
\hline \multicolumn{1}{c}{ Sample } & $\mathrm{N} / \mathrm{Si}$ & $\mathrm{F} / \mathrm{Si}$ & $\mathrm{F} / \mathrm{N}$ \\
\hline 5-fluorouracil & - & - & 0.47 \\
BEA empty & 0 & 0 & - \\
BEA loaded & 0.023 & 0.009 & 0.39 \\
NaX-FAU empty & 0 & $0 *$ & - \\
NaX-FAU loaded & 0.132 & 0.070 & 0.56 \\
\hline
\end{tabular}

"below detection limit 